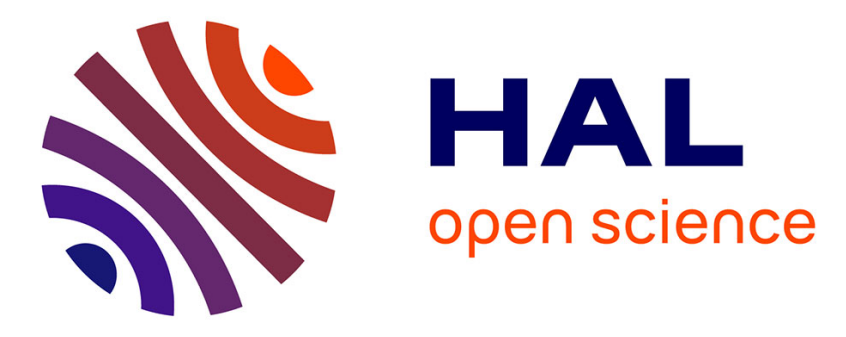

\title{
Detectors for absolute luminosity measurement at DAFNE
}

\author{
G. Mazzitelli, M. Boscolo, F. Bossi, B. Buonomo, F. Murtas, G. Sensolini, P. \\ Raimondi, N. Arnaud, D. Breton, L. Burmistrov, et al.
}

\section{> To cite this version:}

G. Mazzitelli, M. Boscolo, F. Bossi, B. Buonomo, F. Murtas, et al.. Detectors for absolute luminosity measurement at DAFNE. 9th European Workshop on Beam Diagnostics and Instrumentation for Particle Accelerators (DIPAC 2009), May 2009, Basel, Switzerland. pp.345-347. in2p3-00477538

HAL Id: in2p3-00477538

https://hal.in2p3.fr/in2p3-00477538

Submitted on 29 Apr 2010

HAL is a multi-disciplinary open access archive for the deposit and dissemination of scientific research documents, whether they are published or not. The documents may come from teaching and research institutions in France or abroad, or from public or private research centers.
L'archive ouverte pluridisciplinaire HAL, est destinée au dépôt et à la diffusion de documents scientifiques de niveau recherche, publiés ou non, émanant des établissements d'enseignement et de recherche français ou étrangers, des laboratoires publics ou privés. 


\title{
DETECTORS FOR ABSOLUTE LUMINOSITY MEASUREMENT AT DAFNE
}

\author{
G. Mazzitelli", M. Boscolo, F. Bossi, B. Buonomo, F. Murtas, G. Sensolini, P. Raimondi, INFN LNF, \\ Frascati, Italy \\ N. Arnaud, D. Breton, L. Burmistrov, A. Stocchi, A. Variola, B. Viaud, LAL, Orsay, France \\ P. Valente, INFN Roma, Rome, Italy \\ P. Branchini, INFN Roma Tre, Rome, Italy \\ M. Schioppa, INFN Cosenza, Rende, Italy
}

\begin{abstract}
The Frascati electron-positron collider DAFNE is testing the crabbed waist scheme, aiming to reach a large improvement of the specific and integrated luminosity of the accelerator. In order to have a reliable, fast and accurate measurement of the absolute luminosity, a number of dedicated detectors have been designed, built, tested, calibrated and put into operation.
\end{abstract}

\section{INTRODUCTION}

At the Frascati DAFNE accelerator, running at the $\Phi$ meson peak $(\sqrt{ } \mathrm{s}=1.02 \mathrm{GeV})$, the idea of enhancing the luminosity of an electron/positron collider with the introduction of a large Piwinski angle and low vertical beta function compensated by crab waist [1], is being tested. The accelerator has been modified to test the crab waist sextupoles compensation scheme and has restarted operations at the beginning of year 2008. Dedicated detectors have been installed for the measurement of the luminosity and the backgrounds.

\section{Luminosity Detectors}

Three different types of detectors using different physical processes are used, with different degrees of statistical and systematic accuracy:

- resonant decay $e^{+} e^{-} \rightarrow \Phi \rightarrow K^{+} K^{-}$: a set of scintillators (Kaon monitor) has been installed around the vertical axis $\left(\theta \sim 90^{\circ}\right)$ by the SIDDHARTA collaboration to count back-to-back high-ionization tracks: a rate of about $25 \mathrm{~Hz}$ at $10^{32}$ is expected.

- The elastic (Bhabha) scattering $e^{+} e^{-} \rightarrow e^{+} e^{-}$: two calorimetric detectors have been placed on both sides of the interaction region (IR) to detect back-to-back tracks with energy deposit of about the beam energy $\sim 510 \mathrm{MeV}$. Even though the polar angle is limited due to the presence of the low- $\beta$ quadrupoles, the rate expected in the covered acceptance of $18^{\circ} \leq \theta \leq 27^{\circ}$ is high enough $(\sim 440 \mathrm{~Hz}$ at a luminosity of $10^{32} \mathrm{~cm}^{-2} \mathrm{~s}^{-1}$ ) to provide a clean measurement online.

The radiative Bhabha process: $e^{+} e^{-} \rightarrow e^{+} e^{-} \gamma$; it has the advantage of a very high rate and that the emission of highly collimated photons ( $95 \%$ of the signal in contained in a cone of $1.7 \mathrm{mrad}$ aperture), but it is heavily affected by backgrounds. In particular beam losses due to interactions with the residual gas in the beam-pipe,
Touschek effect, and particles at low angles generated close to the IR. Very limited space is available at very small angles, close to the beam pipe, so that compact crystal calorimeters have been realized, in order to count events with a radiated photon.

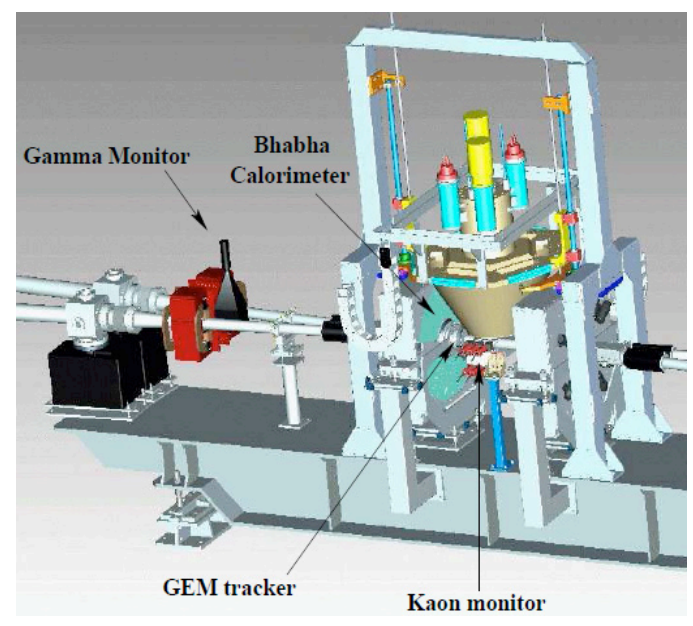

Figure 1: View of the DAFNE interaction region, with the detectors for luminosity measurement around the SIDDHARTA experiment setup.

\section{BHABHA DETECTORS}

The Bhabha monitors consist of two different detectors: a couple of calorimeters and two GEM trackers of annular shape, on either sides of the IR.

\section{Calorimeters}

Two modules of calorimeters surround each of the two final permanent quadrupole magnets, located at a distance of $32.5 \mathrm{~cm}$ on both sides of the IR, as shown in Fig. 1. Each of the four modules are segmented in the azimuth angle in five sectors, $30^{\circ}$ wide. A $1 / 6$ of the acceptance, i.e. the $\pm 15^{\circ}$ region, has not been instrumented, both for leaving space for the supporting structure, and for the high rate of background events expected on the machine plane.

Each sector is a sandwich of 12 trapezoidal tiles of $1 \mathrm{~cm}$ thick scintillator, wrapped with Tyvek, alternated with lead plates: eight $5 \mathrm{~mm}$ thick plates towards the interaction point and three $1 \mathrm{~cm}$ thick plates in the back part, for a total thickness of $19 \mathrm{~cm}$. This choice was 
driven by the compromise between the need of having a good longitudinal containment of $510 \mathrm{MeV}$ electron showers (the total depth corresponds to about $12.5 \mathrm{X}_{0}$ ), and the necessity of having a detector not exceeding the permanent quadrupole length.

Each of the 240 scintillator tiles (produced by injectionmolding in IHEP, Protvino) has three radial grooves on one face, $2 \mathrm{~mm}$ deep (one in the middle and two $1 \mathrm{~cm}$ from the edge of the tile) inside which wavelength shifting (WLS) fibers of $1 \mathrm{~mm}$ diameter are placed; the 36 WLS fibers of a sector are collected to an optical adapter to fit the photocathode of a Photonis-Philips XP 2262B photomultiplier. The assembly of the five sectors of a calorimeter module, before the coupling of fibers to the photomultiplier, is shown in Fig. 2.

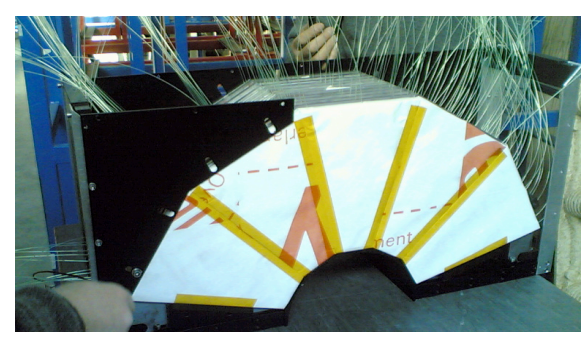

Figure 2: One of the four Bhabha calorimeter modules, each composed by five sectors of 12 scintillator trapezoidal tiles sandwiching 11 lead plates.

The DAQ is based on the KLOE experiment system: the analog signals are actively splitted to be digitized by a constant fraction discriminator for time measurement (using the KLOE TDC, $1.04 \mathrm{~ns}$ resolution), and for the pulse height measurement by the KLOE charge ADC, with a $0.25 \mathrm{pC}$ resolution.

An energy resolution of $15 \%$ at $510 \mathrm{MeV}$ has been measured, before the installation in the IR with a 510 $\mathrm{MeV}$ test-beam at the Frascati BTF, and afterwards with collisions data. This is adequate to define the trigger threshold for selecting Bhabha events. For each of the four modules indeed the analog sum of the five sectors signals is discriminated with a given threshold; then the trigger for a candidate Bhabha event is simply given by the coincidences of two back-to-back modules.

\section{The Triple GEM Trackers}

In front of each calorimeter, at a distance of $18.5 \mathrm{~cm}$ from the IR, a ring of triple-GEM detectors [2] is installed around the beam pipe. The trackers are divided in two units, with an half-moon shape; the top (bottom) half covering azimuth angles between $14^{\circ}$ and $166^{\circ}\left(194^{\circ}\right.$ and $346^{\circ}$ ), as shown in Fig. 3. Each of the four GEM units is segmented into 32 pads: eight cells in azimuth (covering $19^{\circ}$ each) are arranged in four rings of equal radial extension. When a charged particle crosses the $3 \mathrm{~mm}$ drift gap, it generates electrons that will be multiplied by the three GEM foils separated by $2 / 1 / 2 \mathrm{~mm}$. Each of the GEM planes is made of a thin $(50 \mu \mathrm{m})$ kapton foil sandwiched between two copper clads and perforated by a dense set of holes ( $70 \mu \mathrm{m}$ diameter, $140 \mu \mathrm{m}$ pitch).

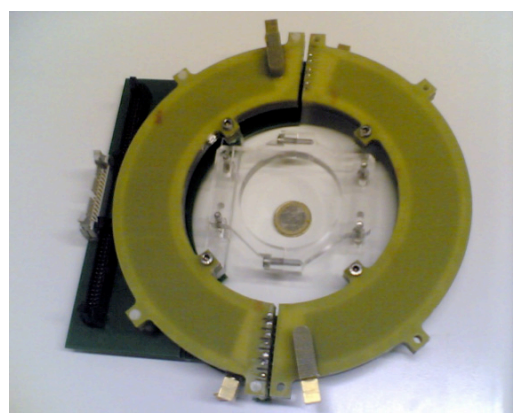

Figure 3: Triple-GEM ring detectors, composing one of the two trackers installed on each side of the IR.

As a high potential difference (about $400 \mathrm{kV}$ ) is applied between the copper sides, the holes act as multiplicating channels and the gain of each layer is about $20(8,000$ in total).

The GEM trackers have been included into the main DAQ system, read by KLOE TDC modules.

\section{RADIATIVE BHABHA DETECTORS}

Two gamma monitor detectors are located $170 \mathrm{~cm}$ away from the IR, collecting the photons radiated by electron or positron beam. Each detector is made of four $\mathrm{PbWO}_{4}$ crystals (squared section of $30 \times 30 \mathrm{~mm}^{2}$ and $110 \mathrm{~mm}$ high) assembled together along $\mathrm{z}$, in order to have a $30 \mathrm{~mm}$ face towards the photon beam, and a total depth of $120 \mathrm{~mm}$ corresponding to about $13 \mathrm{X}_{0}$. Each crystal is readout by a Hamamatsu R7600 compact photomultiplier. Each of the crystal signals is splitted: one half is sent to the charge ADC of the data acquisition system, while the other is sent to an analog mixer. The analog sum of the four crystals is then discriminated and the counts are read by the DAFNE Control system, providing a prompt estimate of the luminosity for machine optimization.

Because of the boost introduced by the beam crossing angle, the trajectories of the photons are shifted towards the inner side (along $\mathrm{x}$ coordinate) of the machine; the gamma monitors and GEM trackers are then placed along the beam pipe at $x=-5 \mathrm{~cm}$ and rotated by $4^{\circ}$ in the horizontal plane with respect to the beam axis.

Thanks to the high rate, those detectors are very useful as a fast feedback for the optimization of machine luminosity versus background, more than providing a measurement of the luminosity, since the relative contribution of background is changing with the machine conditions.

\section{SIMULATION}

In order to get the absolute luminosity from the measured coincidence rate in the Bhabha calorimeters, the event rate should be corrected for the detectors' acceptance and selection efficiency. In order to compute these factors we developed a full simulation of the whole 
experimental set-up, based on the GEANT3 package. This includes all the materials and fields present in the interaction region as well as a simulation of the detectors response.

The BHWIDE package is used to generate Bhabha events with a full treatment of the radiation [3]. The contamination due to the Touschek background is investigated by interfacing an ad hoc generator [4] with the simulation.

The simulation predicts a measured Bhabha event rate of $\sim 440 \mathrm{~Hz}$ when the luminosity equals $10^{32} \mathrm{~cm}^{-2} \mathrm{~s}^{1}$. The rate actually measured at the IP is compared with this number to derive the actual luminosity. The simulation is also used to evaluate the systematic uncertainties affecting this measurement. Using only the calorimeters, an uncertainty of $11 \%$ can be estimated. It drops to $7 \%$ when the GEMs are also used.

We also used the simulation to determine the optimal location for the GEMs. They're shifted in the horizontal plane by $5 \mathrm{~mm}$ in the direction of the boost to compensate for the loss of back-to-back-ness caused by this boost.

\section{RESULTS}

The four calorimeters, the GEM trackers and the crystal gamma detectors are acquired by KLOE farm data acquisition prototype. The trigger condition (T1FREE) consists of the OR of the two possible coincidences of opposite, upside-down modules when the energy released in the modules is above a threshold of about $200 \mathrm{MeV}$.

The precise knowledge of the effective energy threshold in our trigger is essential for the determination of the acceptance with the Monte Carlo simulation. A good estimate of the threshold can be obtained looking at the ratio of the events triggering a given module, with respect to the total acquired events. The threshold profile that one obtains by taking this ratio (bottom plot in Fig. 4) is not a step function due to smearing introduced by the electronics.
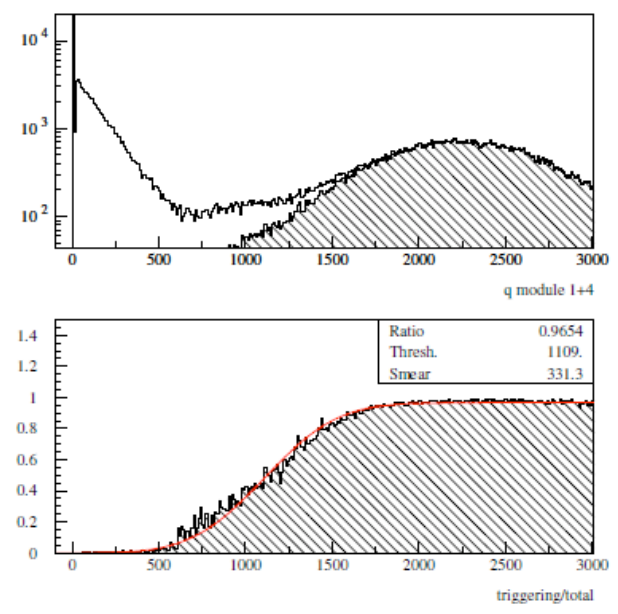

Figure 4: Determination of the trigger threshold by taking the ratio of triggering/total events in one module.
The equivalent energy of the threshold can then be determined from the knowledge of the peak in the energy deposit, which corresponds to the beam energy (510 $\mathrm{MeV}$ ).

Single and coincidence rates are acquired by the DAFNE control system, in order to provide a fast reading of luminosity and background condition very useful for machine parameters optimization.

The T1FREE rate is affected by background contamination, especially during beam injections. In order to get an accurate and clean luminosity estimate, an online filtering process runs on the DAQ farm, providing an offline estimate of the rate (T2FARM), corrected by the measured percentage of background events. This correction is computed analyzing blocks of events (1000 events, corresponding to about $1 \mathrm{~s}$ at $2.5 \times 10^{32}$ ), and by looking at the time distribution of the two triggering modules. In order to isolate genuine Bhabha's, the online filter selects events in around around $\Delta \mathrm{t}=0$ in a $\pm 3 \sigma$ window ( \pm 6 counts). The amount of background beneath the peak is also taken into account: events in the sideband (of the same time width) are counted and subtracted.

All online and filtered data are stored by DAFNE control system with a sampling time of 15 seconds and are available for analysis and on web for online presentation (Fig. 5).

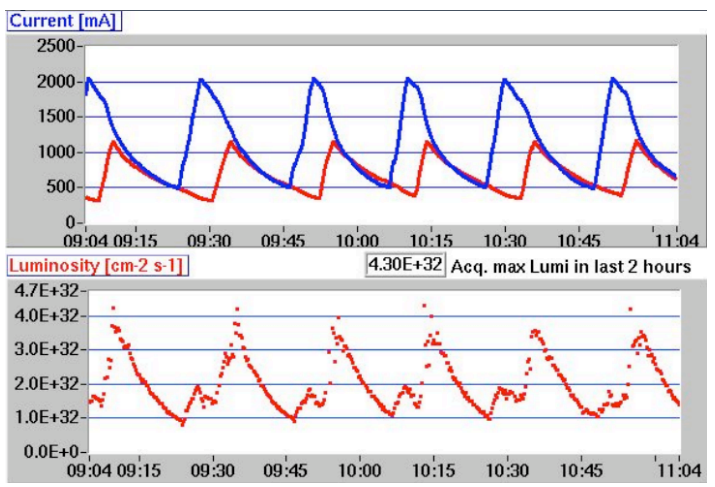

Figure 5: Online display of the currents in the machine (upper plot) and the luminosity measured by our luminometers (bottom plot, including the correction for the acceptance and background subtraction).

\section{ACKNOWLEDGEMENTS}

We would like to thank L. Iannotti, V. Romano and the LNF SSCR service for the mechanical works, and all the electronic staff of the Accelerator Division; G Corradi, M. Pistilli, D. Tagnani for the GEM electronics and detector construction. We also would like to thank W. Placzek for the support on BHWIDE code.

\section{REFERENCES}

[1] D. Alesini et al, LNF-06/033 (IR), 2006.

[2] G. Bencivenni, et al., NIM A488: 493-502, 2002.

[3] S. Jadach et al, Phys.Lett. B390: 298-308, 1997.

[4] M. Boscolo et al, PAC2007, Albuquerque, New Mexico, USA, 2007. 